Pacific Journal of Mathematics

ORDER INTERVALS OF SELFADJOINT LINEAR OPERATORS 


\title{
ORDER INTERVALS OF SELF-ADJOINT LINEAR OPERATORS AND NONLINEAR HOMEOMORPHISMS
}

\author{
E. N. DANCER
}

\begin{abstract}
In this paper, we prove an invertibility criterion for order intervals of self-adjoint linear operators.
\end{abstract}

We then use this to improve and clarify a good deal of recent work (see Bates [2], Bates and Castro [3] and Mawhin [12] for example) on homeomorphism theorems of Ahmad-Lazer type. In particular, we prove a theorem of this type for systems without any conditions on the type of spectrum of the linear part. (Of course, the location of the spectrum is important.) We also show how our ideas can be used to obtain existence results when similar conditions only hold asymptotically under certain regularity assumptions.

Let us describe our main results in more detail. Firstly, we assume that $A$ and $C$ are bounded invertible self-adjoint operators on a Hilbert space $H$ with $A \leq C$. We prove that every self-adjoint operator $B$ with $A \leq B \leq C$ is invertible if and only if $R\left(P_{1}^{+}\right)+R\left(P_{2}^{-}\right)=H$. Here $P_{1}^{+}$is the spectral projection for $A$ corresponding to $(0, \infty), P_{2}^{-}$is the spectral projection for $C$ corresponding to $(-\infty, 0)$ and $R(L)$ denotes the range of $L$. We also obtain a number of equivalent conditions which we need later and we discuss generalizations of the theorem to the case of unbounded operators.

Secondly, we use the above result and a theorem of Browder [5] to obtain a sufficient condition for a nonlinear Gateaux differentiable map $f$ : $H_{1} \subseteq H_{2} \rightarrow H_{2}$ to be a homeomorphism if $f^{\prime}(x)$ is self-adjoint on $H_{2}$ and $A \leq f^{\prime}(x) \leq B$ for $x \in H_{1}$. This uses no compactness assumptions in any form and is in a sense best possible. We also show how linear operators commuting with all the $f^{\prime}(x)$ 's can be used to simplify the verification of our main assumption. In particular, we otain a special case for systems.

Finally, we sketch briefly some applications to partial differential equations.

In $\S 1$, we prove our result on linear operators. In $\S 2$, we prove our result on nonlinear homeomorphisms. Finally, in $\$ 3$, we consider very briefly some applications. 
After our work was completed, we became aware of some work of Amann [1]. Most of our results improve his. Note that there is no analogue of the results in $\$ 1$ in his work. We have shortened our intended list of examples because a number already appeared in his work.

I should like to thank the referee for his careful reading of the manuscript.

1. Invertibility of order intervals of self-adjoints. We assume that $H$ is a real Hilbert space (though the theory would work equally well for complex spaces). Let $S$ denote the set of bounded self-adjoint operators on $H$ and assume that $A, C \in S, A$ and $C$ are invertible and $A \leq C$ (that is, $\langle A x, x\rangle \leq\langle C x, x\rangle$ on $H)$. Let $F=\{B \in S: A \leq B \leq C\}$.

THEOREM 1. The following are equivalent.

(i) Every element of $F$ is invertible.

(ii) There is a connected subset $\mathscr{C}$ of $F$ such that $A \in \mathscr{C}, C \in \mathscr{C}$ and each element of $\mathscr{C}$ is invertible.

(iii) Every element of $F$ is invertible and there is a $q>0$ such that $\left\|B^{-1}\right\| \leq q$ if $B \in F$.

(iv) $R\left(P_{1}^{+}\right)+R\left(P_{2}^{-}\right)=H$, where $P_{1}^{+}$is the spectral projection of $A$ corresponding to $(0, \infty)$ and $P_{2}^{-}$is the spectral projection of $C$ corresponding to $(-\infty, 0)$.

(v) There exist two closed subspaces $M$ and $N$ of $H$ and $a q>0$ such that $H=M+N,\langle A x, x\rangle \geq q\|x\|^{2}$ on $M$ and $\langle C x, x\rangle \leq-q\|x\|^{2}$ on $N$.

Proof. Clearly (iv) $\Rightarrow$ (v), (i) $\Rightarrow$ (ii) and (iii) $\Rightarrow$ (i). It is well known and easy to prove that (v) implies (iii). One simply uses that $A \leq C$ and the assumptions to show that $M \cap N=\{0\}$ and thus $H=M \oplus N$. Hence the corresponding projection is continuous. If $B \in F$, then $B$ is self-adjoint and thus $B$ will be invertible if $\|B x\| \geq q\|x\|$ on $H$. Hence this part of the result will follow if we show that $\|B x\| \geq q\|x\|$ if $x=m+n$ with $m \in M$ and $n \in N$. This is simply proved by showing that $\langle B(m+n), m-n\rangle \geq q\left(\|m\|^{2}+\|n\|^{2}\right)$. A similar argument appears in Mawhin [12]. Thus it remains to show that (ii) implies (iv). This is the major part of the proof. If $B \in \mathscr{C}$, let $P_{B}^{-}$denote the spectral projection of $B$ corresponding to $(-\infty, 0)$. The idea of the proof is to use a continuity argument to show that

$$
R\left(P_{1}^{+}\right)+R\left(P_{B}^{-}\right)=H
$$

for all $B \in \mathscr{C}$. (We first show that it is true if $B=A$.) We now consider the details. If $B=A,(1)$ is true by the spectral theorem (because $\sigma(A)=$ $(\sigma(A) \cap(0, \infty)) \cup(\sigma(A) \cap(-\infty, 0)))$. Assume $D \in \mathscr{C}$. We first show 
that $R\left(P_{1}^{+}\right) \cap R\left(P_{D}^{-}\right)=\{0\}$ and $R\left(P_{1}^{+}\right)+R\left(P_{D}^{-}\right)$is closed. To see this, choose $\varepsilon>0$ such that $\sigma(A) \cup \sigma(D)$ does not intersect $[-\varepsilon, \varepsilon]$. Thus $\sigma\left(\left.A\right|_{R\left(P_{1}^{+}\right)}\right) \subseteq(\varepsilon, \infty)$ and hence

$$
\langle A x, x\rangle \geq \varepsilon\|x\|^{2}
$$

for $x \in R\left(P_{1}^{+}\right)$. Similarly $\langle D x, x\rangle \leq-\varepsilon\|x\|^{2}$ on $R\left(P_{D}^{-}\right)$. Since $A \leq D$, it follows that

$$
\langle A x, x\rangle \leq-\varepsilon\|x\|^{2}
$$

on $R\left(P_{D}^{-}\right)$. We prove that if $m \in R\left(P_{1}^{+}\right), n \in R\left(P_{D}^{-}\right)$and $\|m\|=\|n\|=1$, then $\|m+n\| \geq \varepsilon\|A\|^{-1}$. It follows easily from this that $R\left(P_{1}^{+}\right) \cap R\left(P_{D}^{-}\right)$ $=\{0\}$ and $R\left(P_{1}^{+}\right)+R\left(P_{D}^{-}\right)$is closed. To prove the inequality note that, by the self-adjointness of $A$,

$$
\langle A(m+n), m-n\rangle=\langle A m, m\rangle-\langle A n, n\rangle \geq \varepsilon+\varepsilon=2 \varepsilon
$$

(since $m \in R\left(P_{1}^{+}\right), n \in R\left(P_{D}^{-}\right)$and $\|m\|=\|n\|=1$. Here we have used (2) and (3)). Since

$$
\langle(A(m+n), m-n)\rangle \leq\|A\|\|m+n\|\|m-n\| \leq 2\|A\|\|m+n\|,
$$

the required inequality follows.

Next we show that if $B$ is near $D, R\left(P_{B}^{-}\right)$is close to $R\left(P_{D}^{-}\right)$in the gap sense on $H$ (in the sense of Kato [9, p. 197]). Since $P_{B}^{-}$and $P_{D}^{-}$are projections, this follows easily if we show that $\left\|P_{B}^{-}-P_{D}^{-}\right\| \rightarrow 0$ as $\| B-$ $D \| \rightarrow 0$. This follows from [9, Theorem 6.5.12]. Note that we are assuming that $D$ is invertible.

We have shown that $R\left(P_{1}^{+}\right)+R\left(P_{D}^{-}\right)$is closed, $R\left(P_{1}^{+}\right) \cap R\left(P_{D}^{-}\right)=$ $\{0\}$ and $R\left(P_{D}^{-}\right)$changes continously in the gap sense. By Lemma 4.4.28 in [9], it follows that the codimension of $R\left(P_{1}^{+}\right) \oplus R\left(P_{D}^{-}\right)$is locally constant. Since $\mathscr{C}$ is connected, it follows that the codimension is constant on $\mathscr{C}$. However, we proved that the codimension is zero for $D=A$ and hence it also must be zero for $D=C$, that is, $R\left(P_{1}^{+}\right) \oplus R\left(P_{2}^{-}\right)=H$. This completes the proof.

We now discuss generalizations to the unbounded case. We first need some notation. Assume that $A$ and $C$ are self-adjoint closed operators on $H$ with the same domain $\mathscr{D}(A)$ and $A \leq C$. Let $T=\{B: B$ is closed, $\mathscr{D}(B)=\mathscr{D}(A), A \leq B \leq C\}$. We use the graph norm to topologize $T$. (In other words, $D$ is near $B$ if $B-D$ has small norm as a map of $\mathscr{D}(A)$ with the graph norm into $H$.) Note that $\|x\|+\|B x\|$ is a norm on $\mathscr{D}(A)$ equivalent to the graph norm (cp. [9, p. 191, Remark 1.5]). Then it is 
possible to prove the following partial analogue of Theorem 1. Note that the modulus and square root of self-adjoint operators are studied in [9].

Theorem 1'. Assume that $A$ and $C$ are self-adjoint closed invertible linear operators on $H$ with the same domain and $A \leq C$. In addition, assume that $T$ contains at least one connected set containing both $A$ and $C$. Then the following are equivalent.

(i) Every element of $T$ is invertible.

(ii) There is a connected subset $\mathscr{C}$ of $T$ such that $A \in \mathscr{C}, C \in \mathscr{C}$ and each element of $\mathscr{C}$ is invertible.

(iv) $\mathscr{D}\left(|A|^{1 / 2}\right)=\left(\mathscr{D}\left(|A|^{1 / 2}\right) \cap R\left(P_{1}^{+}\right)\right)+\left(\mathscr{D}\left(|A|^{1 / 2}\right) \cap R\left(P_{2}^{-}\right)\right)$.

(v) There exists subspaces $M$ and $N$ of $\mathscr{D}\left(|A|^{1 / 2}\right)$ closed in the $\mathscr{D}\left(|A|^{1 / 2}\right)$ norm and $q>0$ such that (a) $\mathscr{D}(A) \cap M$ is dense in $M$ for the $\mathscr{D}\left(|A|^{1 / 2}\right)$ norm, (b) $\mathscr{D}(A) \cap N$ is dense in $N$ for the $\mathscr{D}\left(|A|^{1 / 2}\right)$ norm, (c) $M+N=$ $\mathscr{D}\left(|A|^{1 / 2}\right),(\mathrm{d})\langle A x, x\rangle \geq q\|x\|^{2}$ on $M \cap \mathscr{D}(A)$ and $(\mathrm{e})\langle C x, x\rangle \leq-q\|x\|^{2}$ on $N \cap \mathscr{D}(A)$.

REMARKS. This is not really satisfactory for a number of reasons. We do not know if the extra assumption in Theorem $1^{\prime}$ of the existence of the connected set can be avoided. In our applications to nonlinear equations, it is usually trivially satisfied. Note that this extra assumption is only used to prove that (i) $\Rightarrow$ (ii). It certainly holds if $C-A$ is $A$ bounded with $A$ bound less than 1 (in the sense of $[9, \S 4.1 .1]$ ). We simply use

$$
\{A+t(C-A): 0 \leq t \leq 1\} \text {. }
$$

Secondly, it would often be preferable to replace $\mathscr{D}\left(|A|^{1 / 2}\right)$ by $\mathscr{D}(A)$ in (iv) and (v) (with other corresponding changes). This is especially true of (v) where the statement could be simplified. Moreover, $\mathscr{D}\left(|A|^{1 / 2}\right)$ is not always easy to calculate. Unfortunately, we have been unable to prove such a result except if $A$ is bounded below. Note that a density argument shows that the statement corresponding to (iv) with $\mathscr{D}\left(|A|^{1 / 2}\right)$ replaced by $\mathscr{D}(A)$ implies (iv). (A corresponding result holds for (v).) One further problem is that we have no analogue of (iii) of Theorem 1 unless we make further restrictions. I will return to this in a moment.

Sketch of proof of Theorem 1'. The proof has the same basic ideas as the proof of Theorem 1 but the details are more complicated. We need only prove that (ii) $\Rightarrow$ (iv) and (v) $\Rightarrow$ (i). To prove that (v) $\Rightarrow$ (i), we note that, if $B \in T$, then $\mathscr{D}\left(|B|^{1 / 2}\right)=\mathscr{D}\left(|A|^{1 / 2}\right)$ and $|B|^{1 / 2}$ is $|A|^{1 / 2}$ bounded (cp. [9, p. 572]). If (v) holds and $B \in T$, one shows by considering 
$\langle|B|(m+n), m-n\rangle$ that there is a $k>0$ such that

$$
\begin{aligned}
& \left\||B|^{1 / 2}(m+n)\right\|\left\||B|^{1 / 2}(m-n)\right\| \\
& \quad \geq k\left(\|m\|^{2}+\left\||A|^{1 / 2} m\right\|^{2}+\|n\|^{2}+\left\||A|^{1 / 2} n\right\|^{2}\right)
\end{aligned}
$$

if $m \in M \cap \mathscr{D}(A)$ and $n \in N \cap \mathscr{D}(A)$. By a density argument, the same result holds for $m \in M$ and $n \in N$. Since $|B|^{1 / 2}$ is $|A|^{1 / 2}$ bounded, it follows that there is a $\tilde{k}>0$ such that

$$
\left\||B|^{1 / 2} x\right\| \geq \tilde{k}\left(\|x\|+\left\||A|^{1 / 2} x\right\|\right) \geq \tilde{k}\|x\| \quad \text { if } x \in \mathscr{D}\left(|A|^{1 / 2}\right)=M+N .
$$

Hence $|B|^{1 / 2}$ is invertible and thus $B$ is invertible.

To prove that (ii) implies (iv) we use a similar argument to before except that we work in the Banach space $\mathscr{D}\left(|A|^{1 / 2}\right)$. We prove that $\left(R\left(P_{D}^{-}\right) \cap \mathscr{D}\left(|A|^{1 / 2}\right)\right)+\left(R\left(P_{1}^{+}\right) \cap \mathscr{D}\left(|A|^{1 / 2}\right)\right)$ is closed by using an argument similar to that in the proof of the corresponding part of Theorem 1 above except we use an inequality similar to (4) above. (It is here that we run into difficulties if we try to use $\mathscr{D}(A)$ rather than $\mathscr{D}\left(|A|^{1 / 2}\right)$.) Thus the proof can be completed as before if we show that the map $D \rightarrow P_{D}^{-}$ depends continuously on $D$ if $D$ is given the graph norm and $P_{D}^{-}$is considered in the uniform norm as a map of the Banach space $\mathscr{D}\left(|A|^{1 / 2}\right)$ into itself. (The standard reduction theorem ensures that $P_{D}^{-}$commutes with $|D|^{1 / 2}$ and so it maps $\mathscr{D}\left(|D|^{1 / 2}\right)=\mathscr{D}\left(|A|^{1 / 2}\right)$ into itself.) By [9, Theorem 6.5.12], $\left\|P_{D}^{-}-P_{B}^{-}\right\| \rightarrow 0$ as $D \rightarrow B$ in the graph norm. (Note that the norm \|\| is the norm on $H$.) Hence, by the Heinz inequality (cp. [10]), the result will follow if $\left\|P_{B}^{-}-P_{D}^{-}\right\|_{A} \rightarrow 0$ as $D \rightarrow B$. (Here, \|\|$_{A}$ is the usual norm for linear operators on the Banach space $\mathscr{D}(A)$.) Now $B P_{B}^{-}=P_{B}^{-} B$ and $(B+S) P_{D}^{-}=P_{D}^{-}(B+S)$ where $S=D-B$ is small in the graph norm. Hence

$$
\begin{aligned}
B\left(P_{D}^{-}-P_{B}^{-}\right) B^{-1} & =B P_{D}^{-} B^{-1}-P_{B}^{-} \\
& =P_{D}^{-}-P_{B}^{-}+P_{D}^{-} S B^{-1}-S P_{D}^{-} B^{-1} .
\end{aligned}
$$

Now $P_{D}^{-}$commutes with $D$ and hence has norm 1 as a map of $\mathscr{D}(A)=$ $\mathscr{D}(D)$ with the $D$ graph norm into itself. Thus it has locally bounded norm if we put the $B$ graph norm on $\mathscr{D}(A)$ (because the two norms are "nearly the same" if $D$ is near $B$ ). Hence it follows from (5) that $\left\|B\left(P_{D}^{-}-P_{B}^{-}\right) B^{-1}\right\| \rightarrow 0$ as $D \rightarrow B$. The result now follows.

AdDitional REMARKS. I want to return to the question of obtaining estimates for $B^{-1}$. Note that there are three possibilities here. We could try to estimate $B^{-1}$ as a map of $H$ into $H$ or $H$ into $\mathscr{D}\left(|A|^{1 / 2}\right)$ or $H$ into 
$\mathscr{D}(A)$. We briefly discuss all three as they seem to be of interest for nonlinear problems. Firstly, if (v) is strengthened to an assumption on $\mathscr{D}(A)$ (rather than on $\mathscr{D}\left(|A|^{1 / 2}\right)$ ) we easily find that there is a $k>0$ such that $\left\|B^{-1} x\right\| \leq k\|x\|$ if $x \in H$ and $B \in T$. This follows because

$$
\begin{aligned}
\|B(m+n)\|(\|m\|+\|n\|) & \geq\langle B(m+n), m-n\rangle \\
& \geq\langle A m, m\rangle-\langle C n, n\rangle \geq k\left(\|m\|^{2}+\|n\|^{2}\right) .
\end{aligned}
$$

Secondly, if we consider a subset $T_{1}$ of $T$ of those operators for which

$$
\left\||B|^{1 / 2} x\right\| \leq k_{1}\left(\|x\|+\left\||A|^{1 / 2} x\right\|\right)
$$

on $\mathscr{D}\left(|A|^{1 / 2}\right)$ where $k_{1}$ is fixed, we then have a uniform bound for $|B|^{-1 / 2}$ as a map of $H$ into $\mathscr{D}\left(|A|^{1 / 2}\right)$ and hence of $B^{-1}$ as a map of $H$ into $\mathscr{D}\left(|A|^{1 / 2}\right.$ ) for $B$ in $T_{1}$. (One simply uses (4).) Our estimate for $|B|^{1 / 2}$ is used to obtain an upper estimate for $\left\||B|^{1 / 2}(m-n)\right\|$. Note that a condition of the above type holds for each $B \in T$. Our assumption is that we can find a single $k_{1}$ for all $B \in T_{1}$. It seems unlikely that this is always true for $T_{1}=T$. However, we do not have a counterexample. However, it seems to be true in a great many situations. For example, we can take $T_{1}=T$ if $A$ is bounded above or below. Secondly, if there is a $k_{2}>0$ such that

$$
\|B(x)\| \leq k_{2}(\|x\|+\|A x\|)
$$

for $x \in \mathscr{D}(A)$ and $B \in T_{1}$, one can use the Heinz inequality (cp. [10]) to deduce (6) for $B \in T_{1}$. Condition (6) seems to be considerably weaker than (7). Finally, suppose $T_{2}$ is a subset of $T$ and $k_{4}<1<k_{3}$ such that $\|(B-A) x\| \leq k_{3}\|x\|+k_{4}\|A x\|$ for $x \in \mathscr{D}(A)$ and $B \in T_{2}$. Then we have a uniform bound for $B^{-1}$ as a map of $H$ into $\mathscr{D}(A)$ for $B \in T_{2}$. This follows because the results above give a bound for $B^{-1}$ as a map of $H$ into $H$ and because

$$
\|B x\| \geq\left(1-k_{4}\right)\|A x\|-k_{3}\|x\|
$$

for $x \in \mathscr{D}(A)$.

Note that to verify the conditions of Theorem 1(iv) or (v) (or 1'(iv) or $\left.1^{\prime}(\mathrm{v})\right)$ one can use symmetries to simplify the calculations. If $Z$ is self-adjoint and commutes with both $A$ and $C$ and if we have a finite or infinite decomposition of $H, H=\sum R\left(P_{i}\right)$, determined by orthogonal spectral projections $P_{i}$ of $Z$, it suffices to verify (iv) or (v) on each space $R\left(P_{i}\right)$ (since the $P_{i}$ commute with $A$ and $C$ ).

One final comment. Some of our ideas have other uses. The codimension of $R\left(P_{1}^{+}\right)+R\left(P_{D}^{-}\right)$where $D \geq A$ can be used to measure the number 
of eigenvalues of $D$ which cross zero as $D$ varies in cases where $R\left(P_{D}^{+}\right)$and $R\left(P_{D}^{-}\right)$are both infinite dimensional. We return to this briefly in $\S 2$.

2. Nonlinear homeomorphisms. We apply the results of the previous sections to nonlinear maps. We first consider the bounded case.

Theorem 2. Assume that $A$ and $C$ are self-adjoint bounded invertible linear operators on $H$, that $f: H \rightarrow H$ is Gateaux-differentiable for every $x \in H$ and that $A \leq f^{\prime}(x) \leq C$ and $f^{\prime}(x)$ is self-adjoint for $x \in H$.

(i) If $R\left(P_{1}^{+}\right)+R\left(P_{2}^{-}\right)=H$, then $f$ is a homeomorphism of H. Here $P_{1}^{+}\left(P_{2}^{-}\right)$is the spectral projection for $A(C)$ corresponding to $(0, \infty)$ $((-\infty, 0)$.

(ii) If $f^{\prime}(x)$ is invertible for $x \in H$, if there exist $x_{0}, y_{0} \in H$ such that $f^{\prime}\left(x_{0}\right)=A$ and $f^{\prime}\left(y_{0}\right)=C$ and if there is a subset $\tilde{T}$ of $H$ containing $x_{0}$ and $y_{0}$ such that $f^{\prime}(\tilde{T})$ is connected, then $f$ is a homeomorphism.

Proof. Part (ii) follows from part (i) by using that (ii) implies (iv) in Theorem 1. (Set $\left.C=f^{\prime}(\tilde{T})\right)$. To prove (i), note that

$$
\left\|f^{\prime}(x)\right\| \leq \sup \{\|A\|,\|C\|\} \quad \text { for } x \in H
$$

and thus

$$
\|f(x)-f(y)\| \leq \sup \{\|A\|,\|C\|\}\|x-y\| \text { for } x, y \in H .
$$

Hence $f$ is continuous. Assume that, for each $x, y \in H$, there is a self-adjoint linear operator $D(x, y)$ on $H$ such that $A \leq D(x, y) \leq C$ and

$$
f(x)-f(y)=D(x, y)(x-y) .
$$

We will return to this at the end of the proof. Since (iv) implies (iii) in Theorem 1 , we see that $D(x, y)$ is invertible and there is a $q>0$ such that $\left\|(D(x, y))^{-1}\right\| \leq q$ for $x, y \in H$. Hence, by (8), $\|f(x)-f(y)\| \geq$ $q^{-1}\|x-y\|$ for $x, y \in H$. Since $f$ is continuous, it follows easily from this result that $f$ has closed range. Since $f$ is Gateaux-differentiable and $f^{\prime}(x)$ is invertible for $x \in H$, it follows from Browder [5, Theorem 2] that $f$ is onto. That $f$ is a homeomorphism now follows easily.

It remains to prove the existence of $D(x, y)$. The obvious candidate for $D(x, y)$ is $\tilde{D}$ where $\tilde{D}$ is defined by $\tilde{D} u=\int_{0}^{1} f^{\prime}(x+t(y-x)) u d t$. It is easy to check that this has all the right properties provided we prove that the integral exists. Since our assumptions give a bound on $f^{\prime}$, it suffices to prove the measurability of the integrand. This follows because the integrand is the pointwise limit of continuous functions (by [7, 
Corollary III.6.14]). In fact,

$$
s^{-1}(f(x+t(y-x)+s u)-f(x+t(y-x))) \rightarrow f^{\prime}(x+t(y-x)) u
$$

as $s \rightarrow 0$. This completes the proof.

We now want to consider the unbounded case. We start with the simplest generalization which applies in many cases. Assume that $\tilde{A}$ is a closed self-adjoint operator on $H$ with domain $\mathscr{D}(\tilde{A})$ and $f: \mathscr{D}(\tilde{A}) \rightarrow H$ is Gateaux-differentiable (where $\mathscr{D}(\tilde{A})$ has the graph norm) such that each $f^{\prime}(x)$ is closed and self-adjoint on $H$ and such that $A \leq f^{\prime}(x) \leq C$ on $H$, where $A$ and $C$ are closed self-adjoint operators with the same domain as $\tilde{A}$. Assume that $\mathscr{D}\left(|\tilde{A}|^{1 / 2}\right)=\left(\mathscr{D}\left(|\tilde{A}|^{1 / 2}\right) \cap R\left(P_{1}^{+}\right)\right) \cup\left(\mathscr{D}\left(|\tilde{A}|^{1 / 2}\right) \cap R\left(P_{2}^{-}\right)\right)$. Finally, assume that there exist $k_{3}<1<k_{4}$ such that

$$
\left\|f^{\prime}(x) y-\tilde{A} y\right\| \leq k_{3}\|\tilde{A} y\|+k_{4}\|y\|
$$

for $x, y \in \mathscr{D}(\tilde{A})$. Then $f$ is a homeomorphism of $\mathscr{D}(\tilde{A})$ onto $H$. The proof of this is an easy modification of the above proof if one uses Theorem $1^{\prime}$ and the comments after it. The fairly restrictive assumption (9) is used twice. Firstly, it is used to show that $f$ is continuous as a map on $\mathscr{D}(\tilde{A})$ into $H$ and that $f^{\prime}(x+t(y-x)) u$ is bounded on [0,1]. However, these conditions hold under much weaker assumptions or could be assumed. Much more importantly, it is used to obtain a uniform bound of $D(x, y)^{-1}$ as a map of $H$ into $\mathscr{D}(\tilde{A})$ and hence to deduce that $f$ has closed range. However, many variants could be proved. For example, if we have an a priori bound for $x$ in $\mathscr{D}(\tilde{A})$ when $f(x)$ is bounded, it suffices to assume (9) for $x$ in a bounded set in $\mathscr{D}(\tilde{A})$. Secondly, if we assume (9) with any $k_{3}$ and $k_{4}$ or if the $f^{\prime}(x)$ 's are uniformly bounded below, then our earlier comments on the use of $\left|f^{\prime}(x)\right|^{1 / 2}$ imply that $D(x, y)^{-1}$ is uniformly bounded as a map of $H$ into $\mathscr{D}\left(|\tilde{A}|^{1 / 2}\right)$. It follows that if $f\left(x_{n}\right) \rightarrow y$ then $\left\{x_{n}\right\}$ is Cauchy in $\mathscr{D}\left(|\tilde{A}|^{1 / 2}\right)$. Hence, if $f(x)=L x+r(x)$ where $L$ is linear and closed and $\mathscr{D}(L)=\mathscr{D}(\tilde{A})$ and if $r$ extends to a continuous map of $\mathscr{D}\left(|\tilde{A}|^{1 / 2}\right)$ into $H$, then $x_{n}$ converges in $\mathscr{D}(\tilde{A})$. It follows that $f$ has closed range and we obtain another variant of Theorem 2 . The second part of Theorem 2 can be similarly generalized.

Additional remarks. (These apply to both the bounded and unbounded case.)

1. In applications, the assumption that $f^{\prime}(\tilde{T})$ is connected seems to usually cause little difficulty. One can usually find rather "nice" elements $x_{0}, y_{0}$ in $\mathscr{D}(\tilde{A})$ such that $f^{\prime}\left(x_{0}\right)=A, f^{\prime}\left(y_{0}\right)=C$ and such that the map $t \rightarrow f^{\prime}\left(t x_{0}+(1-t) y_{0}\right)$ is continuous even thouugh the map $x \rightarrow f^{\prime}(x)$ is not continuous. 
2. Part (ii) of Theorem 2 is in a sense best possible. Indeed suppose that (i) $f: \mathscr{D}(\tilde{A}) \rightarrow H$ is (Fréchet) twice continuously differentiable, (ii) there is a connected subset $\tilde{\mathscr{T}}$ of $\mathscr{D}(A), \tilde{x}_{0}, \tilde{y}_{0} \in \tilde{\mathscr{T}}$ and an invertible closed self-adjoint linear operator $A_{1}$ with domain $\mathscr{D}(\tilde{A})$ such that $f^{\prime}(x) \geq A_{1}$ for $x \in \tilde{\mathscr{T}}$, (iii) $f^{\prime}(x)$ is Fredholm for $x \in \tilde{\mathscr{T}}$, (iv) $f^{\prime}\left(\tilde{x}_{0}\right)$ and $f^{\prime}\left(\tilde{y}_{0}\right)$ are both invertible and $(\mathrm{v})\left(\mathscr{D}\left(|\tilde{A}|^{1 / 2}\right) \cap R\left(\tilde{P}_{1}^{+}\right)\right)+\left(\mathscr{D}\left(|\tilde{A}|^{1 / 2}\right) \cap R\left(P_{x}^{-}\right)\right)$has different codimensions at $x=\tilde{x}_{0}$ and $x=\tilde{y}_{0}$. Then $f$ is not a local homeomorphism at some point of $\tilde{\mathscr{T}}$. (Here $\tilde{P}_{1}^{+}$is the spectral projection for $A_{1}$ corresponding to $(0, \infty)$ and $P_{x}^{-}$is the spectral projection for $f^{\prime}(x)$ corresponding to $(-\infty, 0)$.) In our case, it is usually natural to set $A_{1}=A$ and $\tilde{x}_{0}=x_{0}$. The only problem is that in many of the natural applications (for example, nonlinear wave equations) $f$ is probably not $C^{2}$. The above result is proved by using our continuation ideas (as mentioned at the end of $\S 1$ ) to show that an eigenvalue $\lambda(x)$ of $f^{\prime}(x)$ (where $\lambda(x)$ depends continuously on $x$ ) changes sign on $\tilde{\mathscr{T}}$ and by then applying the main result in [6]. If each $f^{\prime}(x)$ is bounded below and if $f^{\prime}(x)-\lambda I$ is Fredholm on $(-\infty, 0]$, the results in [6] apply much more directly.

We now consider a special case of Theorem 2 which in fact motivated our work.

THEOREM 3. Assume that $J$ and $K$ are self-adjoint matrices on $R^{n}$ such that $J \leq K$. Assume that $L: D(L) \subseteq H^{n} \rightarrow H^{n}$ is self-adjoint and closed and $L$ commutes with $\mathscr{J}$ and $\mathscr{K}$ where $\mathscr{J}$ is the natural map on $H^{n}$ induced by $J$ and $\mathscr{K}$ is defined analogously. Let $a_{t}\left(b_{l}\right)$ denote the eigenvalues of $J(K)$ written in increasing order and counting multiplicity. Assume that $\left[a_{i}, b_{i}\right] \cap$ $\sigma(L)=\varnothing$ for $1 \leq i \leq n$ and that $F: \mathscr{D}(L) \rightarrow H^{n}$ is Gateaux-differentiable such that $F^{\prime}(x)$ is symmetric on $\mathscr{D}(L)$ and $\mathscr{J} \leq F^{\prime}(x) \leq \mathscr{K}$ for $x \in \mathscr{D}(L)$. Then $L-F$ is a homeomorphism of $\mathscr{D}(L)$ and $H$.

Proof. We apply Theorem 2(i) and the comments after Theorem2. We set $f(x)=L x-F(x), A=L-\mathscr{K}$ and $C=L-\mathscr{J}$. Note that, since $\mathscr{J}$ and $\mathscr{K}$ are bounded, $F^{\prime}(x)$ extends to a bounded self-adjoint linear operator on $H^{n}$. Moreover, since $\mathscr{J} \leq F^{\prime}(x) \leq \mathscr{K}$,

$$
\left\|F^{\prime}(x)\right\| \leq \sup \{\|\mathscr{J}\|,\|\mathscr{K}\|\} .
$$

As in the proof of Theorem 2, it follows that $F$ is a continuous map of $\mathscr{D}(L)$ into $H$. The result will follow from the comments after Theorem 2, if we show that

$$
\mathscr{D}\left(|L|^{1 / 2}\right)=\left(\mathscr{D}\left(|L|^{1 / 2}\right) \cap R\left(P_{1}^{+}\right)\right) \cup\left(\mathscr{D}\left(|L|^{1 / 2}\right) \cap R\left(P_{2}^{-}\right)\right) .
$$


Let $\left\{\hat{T}_{i}\right\}_{i=0}^{k}$ denote the components of $R \backslash \bigcup_{i=1}^{n}\left[a_{l}, b_{l}\right]$, where $\hat{T}_{0}$ is the component "containing" $-\infty$ and $\hat{T}_{k}$ is the component "containing" $+\infty$. Let $P_{i}$ be the spectral projection for $L$ corresponding to $\hat{T}_{i}$. By our assumption on $\sigma(L), \sum_{i=0}^{k} P_{i}=I$. Since $L$ commutes with $\mathscr{J}, \mathscr{J}$ commutes with $P_{i}$. Hence $A$ and $C$ commute with $P_{i}$. Since the $P_{l}$ all commute and they each commute with $|L|^{1 / 2}$, it follows easily that it suffices to establish (10) on each subspace $R\left(P_{i}\right)$. For $i=0$, we note that $\langle L x, x\rangle \leq \mu(x, x)$ on $R\left(P_{0}\right) \cap \mathscr{D}(L)$, where $\mu=\sup \left(\sigma(L) \cap\left(-\infty, a_{1}\right)\right)<a_{1}$. Since $J \geq a_{1} I$, $\mathscr{J} \geq a_{1} I$ and hence $\langle\mathscr{J} x, x\rangle \geq a_{1}(x, x)$ on $H^{n}$. Thus $\langle C x, x\rangle \leq$ $\left(\mu-a_{1}\right)(x, x)$ on $R\left(P_{0}\right)$ and hence $C$ is strongly negative definite on $R\left(P_{0}\right)$. Hence $R\left(P_{2}^{-}\right) \cap R\left(P_{0}\right)=R\left(P_{0}\right)$. Similarly, $R\left(P_{1}^{+}\right) \cap R\left(P_{k}\right)=$ $R\left(P_{k}\right)$. Hence we need only consider $R\left(P_{i}\right)$ where $1 \leq i \leq k-1$. Thus we are now in the case where $L$ is bounded. Let $x_{i}=\inf \left(\sigma(L) \cap \hat{T}_{l}\right)$ and $y_{i}=\sup \left(\sigma(L) \cap \hat{T}_{i}\right)$. By our assumption on $\sigma(L)$, there is a $j$ such that $b_{j-1}<x_{i}<y_{i}<a_{j}$. Let $T_{i}$ denote the spectral projection on $R^{n}$ corresponding to the eigenvalues less than or equal to $b_{j-1}$ for $K$ and let $\tilde{T}_{l}$ be the spectral projection on $R^{n}$ corresponding to the eigenvalues of $J$ in $\left[a_{j}, \infty\right)$. One easily sees that $R^{n}=R\left(T_{i}\right) \oplus R\left(\tilde{T}_{i}\right)$. This idea is due to Lazer [11]. Let $P$ be the corresponding projection onto $R\left(T_{i}\right)$. Let $\mathscr{P}$ be the projection on $H^{n}$ induced by $P$. Assume that $\mathscr{P}$ commutes with the $P_{i}$. (We will prove this in a moment.) It follows that $\mathscr{P}$ maps $R\left(P_{l}\right)$ into itself. In particular, $R\left(P_{i}\right)=\left(R(\mathscr{P}) \cap R\left(P_{i}\right)\right) \oplus\left(R(I-\mathscr{P}) \cap R\left(P_{i}\right)\right)$ (where we are thinking of $\mathscr{P}$ as a map only on $R\left(P_{i}\right)$ ). Since $R(P)=R\left(T_{i}\right)$, it follows easily that $R(\mathscr{P})=R\left(\mathscr{T}_{t}\right)$, where $\mathscr{T}_{i}$ is the projection on $H^{n}$ induced by $T_{l}$. (We are using here that the map $S \rightarrow \mathscr{S}$ from an $n \times n$ matrix to the induced operator on $H^{n}$ is a ring homomorphism.) It is easy to check that $\mathscr{T}_{l}$ is the spectral projection corresponding to $\left(-\infty, b_{J-1}\right]$ for $\mathscr{K}$. We prove that $A$ is strongly positive on $R(\mathscr{P}) \cap R\left(P_{i}\right)$. Since $\sigma\left(\left.L\right|_{R\left(P_{i}\right)}\right) \subseteq$ $\left[x_{i}, y_{i}\right],\langle L x, x\rangle \geq x_{i}\langle x, x\rangle$ on $R\left(P_{i}\right)$. Moreover, since $\sigma\left(\left.K\right|_{R\left(T_{i}\right)}\right)$ $\subseteq\left(-\infty, b_{j-1}\right],\langle K x, x\rangle \leq b_{j-1}\langle x, x\rangle$ on $R\left(T_{i}\right)$. Hence $\langle A x, x\rangle \geq$ $\left(x_{i}-b_{j-1}\right)\langle x, x\rangle$ on $R(\mathscr{P}) \cap R\left(P_{i}\right)=R\left(\mathscr{T}_{i}\right) \cap R\left(P_{i}\right)$. Since $\left.x_{t}-b_{J-1}\right\rangle$ 0 , this proves the positivity. Similarly $\langle C x, x\rangle \leq\left(y_{i}-a_{j}\right)\langle x, x\rangle$ on $R(I-\mathscr{P}) \cap R\left(P_{i}\right)$. This gives the required decomposition. Note that, by using Theorem $1^{\prime}(\mathrm{iv})$ rather than Theorem $1^{\prime}(\mathrm{v})$, we can avoid showing that the subspace we have constructed is the range of the spectral projection though this is not difficult to show.

It remains to show that $\mathscr{P}$ commutes with the $P_{i}$. Now it is easy to show that a bounded linear operator $S$ commutes with a projection $\tilde{P}$ if and only if $S$ leaves both $N(\tilde{P})$ and $R(\tilde{P})$ invariant. Thus it suffices to prove that $P_{i}$ maps $R(\mathscr{P})=R\left(\mathscr{T}_{i}\right)$ and $N(\mathscr{P})=R\left(\tilde{\mathscr{T}}_{l}\right)$ into themselves, 
where $\tilde{\mathscr{T}}_{i}$ is the projection induced by $\tilde{T}_{l}$. This follows because $J$ commutes with $L$ and hence their spectral projections commute.

If one examines the above arguments, one can show that the assumption on $\sigma(L)$ is the best possible one to ensure our main condition on spectral projections when $A$ and $C$ are invertible. The non-uniqueness results mentioned earlier could also be applied in the above case. Note that the assumption that $L$ commutes with $\mathscr{J}$ and $\mathscr{K}$ certainly holds if $L$ is of the form $L\left(u_{1}, \ldots, u_{n}\right)=\left(Z u_{1}, \ldots, Z u_{n}\right)$, where $Z$ is a self-adjoint operator on $H$ but it holds more generally. (For example, it holds if $J$ and $K$ are diagonal matrices and $L$ is a diagonal operator on $H^{n}$.) Theorem 3 improves a result of Amann [1] by placing no condition on $L$ (except that $\left[a_{i}, b_{l}\right] \cap \sigma(L)=\varnothing$ for $\left.1 \leq i \leq n\right)$ and by weakening the commutativity requirements.

It is possible to sometimes get results for the unbounded case by looking at a related bounded problem and obtaining generalized solutions. There are several ways of doing this. We assume the situation in the comments on the unbounded case after the proof of Theorem 2. Assume that $|\tilde{A}|^{-1} f(x)$ extends to a Gâteaux-differentiable map $W(x)$ of $\mathscr{D}\left(|\tilde{A}|^{1 / 2}\right)$ into itself. (Now $\mathscr{D}\left(|\tilde{A}|^{1 / 2}\right.$ ) is a Hilbert space for the scalar product $\langle u, v\rangle_{1}=\left\langle|\tilde{A}|^{1 / 2} u,|\tilde{A}|^{1 / 2} v\right\rangle$. Thus, if $x \in \mathscr{D}(\tilde{A}), W(x)$ is characterized by $\langle W(x), v\rangle_{1}=\langle f(x), v\rangle$. This tends to be the easiest way to understand the extension.) We now use our theory for the bounded case to ask if $W$ is a homeomorphism of $\mathscr{D}\left(|\tilde{A}|^{1 / 2}\right)$. Note that, if $x \in \mathscr{D}(\tilde{A}), f(x)=h$ if and only if $W(x)=|\tilde{A}|^{-1} h$. Thus asking if $W$ is a homeomorphism is asking for generalized solutions of the equation for $h$ in a more general space than $H$ (and the corresponding continuity properties). This generalized formulation is sometimes convenient and sometimes not. It seems probable that the spectral assumptions for the two methods to be applicable are similar but not quite equivalent in the case of continuous spectra. If one can prove that the generalized solutions are more regular, one can often deduce that $f$ is a homeomorphism (if $W$ is). Note that the above method of looking for generalized solutions is a generalization of looking for solutions in the Sobolev space $W^{1,2}$ (instead of $W^{2,2}$ ) for second order elliptic problems.

Finally, our methods can sometimes be used to establish a priori bounds for solutions of equations which do not satisfy the assumptions of Theorem 2 but satisfy them asymptotically. Thus if we have a map $\mathscr{A}(x)$ such that $\mathscr{A}(x)=f(x)+r(x)$ for large $x$ where $f$ satisfies the assumptions of Theorem 2 and $\|x\|^{-1} r(x) \rightarrow 0$ as $\|x\| \rightarrow \infty$, we can deduce a bound for $x$ if $\mathscr{A}(x)=y$ (since $\|f(x)\| \geq k\|x\|)$. This can sometimes be 
combined with degree type arguments to prove that $\mathscr{A}$ is onto (as in Mawhin and Ward [13]). Another example appears in \$3. Of course, this method needs stringent assumptions on $\mathscr{A}$ to ensure that some form of degree theory can be used.

3. Some examples. Probably the most interesting applications of Theorem 3 are to nonlinear wave equations. However, since this was discussed in [1], we content ourselves with discussing very briefly some other examples. (Note, however, that we could sometimes improve the results in [1] by allowing a slightly more general linear term.) We assume a knowledge of basic properties of elliptic operators as in Friedman [8].

Firstly, we consider the problem

$$
\Delta^{2} u+\frac{\partial}{\partial x}\left(g\left(\frac{\partial u}{\partial x}\right)\right)=f(x)
$$

in $\Omega$ with boundary conditions $u=\Delta u=0$ on $\partial \Omega$, where $\Omega$ is a smooth bounded domain in $R^{n}$. Assume that $g$ is differentiable and $c \leq g^{\prime}(y) \leq d$ on $R$. Assume that zero is not an eigenvalue of $\Delta^{2} u+a\left(\partial^{2} u / \partial x^{2}\right)$ (with the boundary conditions) for any $a$ in $[c, d]$. This is an example where it is best to first work with generalized solutions on the space $\mathscr{D}\left(\left(\Delta^{2}\right)^{1 / 2}\right)$ where $\Delta^{2}$ includes the boundary conditions. It is well-known and easy to prove that $\left(\Delta^{2}\right)^{1 / 2}$ is simply $-\Delta$ with Dirichlet boundary conditions. On $\mathscr{D}(\Delta)$, our equation becomes $u+\mathscr{A}(u)=\left(\Delta^{2}\right)^{-1} f$, where $\mathscr{A}$ is defined on $\mathscr{D}(\Delta)$ by

$$
\langle\mathscr{A}(u), v\rangle_{D(\Delta)}=-\left\langle g\left(\frac{\partial u}{\partial x}\right), \frac{\partial v}{\partial x}\right\rangle
$$

It is easy to prove using the embedding theorems that $\mathscr{A}$ is a Gateaux-differentiable map of $\mathscr{D}(\Delta)$ into itself. Our operators $A$ and $C$ are simply the operators

$$
I+\Delta^{-2}\left(d \frac{\partial^{2} u}{\partial x^{2}}\right), \quad I+\Delta^{-2}\left(c \frac{\partial^{2} u}{\partial x^{2}}\right)
$$

respectively. Now it is easily checked that

$$
I+\Delta^{-2}\left(r \frac{\partial^{2} u}{\partial x^{2}}\right)
$$

is a compact linear operator and thus the spectrum consists only of eigenvalues with eigenfunctions in $\mathscr{D}\left(\Delta^{2}\right)$ (by standard regularity theory). 
Hence

$$
I+\Delta^{-2}\left(r \frac{\partial^{2} u}{\partial x^{2}}\right)
$$

is invertible on $\mathscr{D}(\Delta)$ if $c \leq r \leq d$ by our assumptions. By Theorem $1, B$ is invertible if $A \leq B \leq C$. Hence, by Theorem $2, I+\mathscr{A}$ is a homeomorphism of $\mathscr{D}(\Delta)$ into itself. In other words, for each $f \in Z$, (11) has a unique generalized solution and the inverse map is continuous as a map of $Z$ into $\mathscr{D}(\Delta)$. Here $Z$ denotes $\left\{\Delta u: u \in \dot{L}^{2}\right\}$ with the natural norm and our derivatives are distributional derivatives. If $u \in \mathscr{D}(\Delta)$, then by Morrey [14, p. 66] $(\partial / \partial x) g(\partial u / \partial x) \in L^{2}$. If $u+\mathscr{A}(u)=f$ and $f \in L^{2}$, it follows that $u \in \mathscr{D}\left(\Delta^{2}\right)$. Thus $u$ is a strong solution (that is, a solution in $W^{4,2}(\Omega)$ ). Provided that $g^{\prime}$ is continuous (though this could be weakened), it is easy to show that the map $(\partial / \partial x) g(\partial u / \partial x)$ is a continuous map of $\mathscr{D}(\Delta)$ into $L^{2}$. It follows easily that the inverse map (for equation (11)) is continuous as a map of $L^{2}$ into $\mathscr{D}\left(\Delta^{2}\right)$ as required. If we tried to treat this example directly on $\mathscr{D}\left(\Delta^{2}\right)$, it seems that our earlier comments on the unbounded case only apply under additional assumptions (and even then some care is needed). This illustrates how the reduction to the bounded case is often useful. It also illustrates how the various equivalences in Theorem 1 can be used to simplify statements of theorems. Finally, the above could be used with slight modifications if we change the boundary conditions on $\Delta^{2}$ to Dirichlet boundary conditions. (We now do not have a simple formula for $\left(\Delta^{2}\right)^{1 / 2}$ but this does not matter.) Moreover, by using degree theory together with the present ideas, it can be shown that the unique solvability still holds if $g$ is merely Lipschitz (instead of differentiable).

As our last example, we consider a system of one-dimensional wave equations. Assume that $G$ is a $C^{2}$ convex function on $R^{n}$. We consider the problem

$$
\tilde{\square} u=\nabla G(u)-f(x, t)
$$

in $\Omega=[0,2 \pi] \times[0, \pi]$ where $u=\left(u_{1}, \ldots, u_{n}\right)$, $\tilde{\square}$ denotes the diagonal operator each of whose components is $\square u=u_{t t}-u_{x x}$ with boundary conditions $u(2 \pi, x)=u(0, x), u_{t}(2 \pi, x)=u_{t}(0, x), u(t, 0)=u(t, \pi)=0$. (As in [12], the boundary conditions only hold in a generalized sense.) We assume that there exist self-adjoint matrices $J$ and $K$ such that $\left[a_{l}, b_{i}\right] \cap$ $\sigma(\square)=\varnothing$ for $1 \leq i \leq n$, where $a_{i}\left(b_{l}\right)$ denote the eigenvalues of $J(K)$ in increasing order and counting multiplicity. We assume that there is an $r>0$ such that if $y \in R^{n}$ and $\|y\| \geq r$, then $\nabla G(y)=\omega(y) y+\bar{f}(y)$, 
where $\omega(y)$ is a self-adjoint linear map on $R^{n}, \omega(y)$ depends continuously on $y, J \leq \omega(y) \leq K$ and $\|y\|^{-1} \bar{f}(y) \rightarrow 0$ as $\|y\| \rightarrow \infty$. This holds if $\nabla G$ is asymptotically linear with $J \leq(\nabla G)^{\prime}(\infty) \leq K$ but it holds in other cases. We extend $\omega$ to a continuous map of $R^{n}$ into $\tilde{Z}$, the self-adjoint matrices $\tilde{C}$ with $J \leq \tilde{C} \leq K$. (We can do this because $\tilde{Z}$ is contractible.) It is then easy to prove that

$$
\nabla G(u)-\omega(u) u=\tilde{f}(u)
$$

for $u$ in $L^{2}(\Omega)^{n}$, where $\|\tilde{f}(u)\|_{2} /\|u\|_{2} \rightarrow 0$ as $\|u\|_{2} \rightarrow \infty$. Here \|\|$_{2}$ denotes the usual norm on $L^{2}(\Omega)^{n}$. (One splits $\Omega$ into $\{x:|u(x)| \geq r\}$ and its complement.) If $\varepsilon>0$, consider the equation

$$
\tilde{\square} u=\nabla G(u)+\varepsilon u-f .
$$

Then $\tilde{\square} u-(\omega(u)+\varepsilon I) u=\tilde{f}(u)-f$. Since $J \leq \omega(y) \leq K$, then $\mathscr{J} \leq$ $\omega(u)+\varepsilon I \leq \mathscr{K}+\varepsilon I$. Here, as in $\S 2, \mathscr{J}$ denotes the mapping on $L^{2}(\Omega)^{n}$ generated by $J$ and $\mathscr{K}$ is defined analogously. Since the assumptions of Theorem 3 are stable to small perturbations, we can argue as in the proof of Theorem 3 to deduce that there exist $\varepsilon_{0}, k>0$ such that

$$
\|\tilde{\square} u-\omega(u)-\varepsilon u\|_{2} \geq k\|u\|_{2}
$$

if $0 \leq \varepsilon \leq \varepsilon_{0}$ and $u \in \mathscr{D}(\tilde{\square})$. It follows easily that there is an $M>0$ such that $\|u\|_{2} \leq M$ if $u$ is a solution of (13) with $0 \leq \varepsilon \leq \varepsilon_{0}$. Thus we have an a priori bound.

We now prove that, if (13) is solvable for every $\varepsilon>0$, then it is also solvable for $\varepsilon=0$. Suppose $u^{\varepsilon}$ is a solution for each $\varepsilon>0$. We can write the components $u_{i}^{\varepsilon}=v_{i}^{\varepsilon}+w_{l}^{\varepsilon}$, where $v_{i}^{\varepsilon} \in N(\square)$ and $w_{i}^{\varepsilon} \in N(\square)^{\perp}$. Since 미 $\left.\right|_{N(\square)^{\perp}}$ has compact inverse (cp. [4] or [12]) and since $\left\|u^{\varepsilon}\right\|_{2} \leq M$ for all $\varepsilon$, it follows easily from the equation satisfied by $w_{i}^{\varepsilon}$, that $\left\{w_{l}^{\varepsilon}\right\}_{0<\varepsilon \leq \varepsilon_{0}}$ is compact in $L^{2}(\Omega)$. (It is at this stage that we use the fact that we have one-dimensional wave equations.) Thus, by choosing a subsequence if necessary, we can ensure that $w_{i}^{\varepsilon} \rightarrow w_{i}^{0}$ strongly, $u_{i}^{\varepsilon} \rightarrow u_{i}^{0}$ weakly and $\nabla G\left(u^{\varepsilon}\right) \rightarrow z$ weakly as $\varepsilon \rightarrow 0$. We can then use the Minty trick to complete the proof. (A similar argument appears in [4] or [12].) It is here that we use $G^{\prime}$ is monotone.

Thus, to prove that (12) has a solution, it suffices to solve (13) for $0<\varepsilon \leq \varepsilon_{0}$. Let $P$ be the orthogonal projection onto $N(\tilde{\square})$. The equation $P(\nabla G(v+w)+\varepsilon(v+w)-f)=0$ (where $v \in N(\tilde{\square})$ and $w \in N(\tilde{\square})^{\perp}$ ) can be uniquely solved for $v$ as a function of $w$. One way to see this is to note that, since $(\nabla G)^{\prime}(y) \geq 0$ for $y \in R^{n}$, the Gateaux derivative $T(v, w)$ (in $v$ ) of the left hand side of this equation satisfies $T(v, w) \geq \varepsilon I$ for all $v$, $w$. One can then use Browder's theorem as in $\$ 2$. (Alternatively one can 
use monotonicity.) Moreover, it is well-known and easy to prove that the unique solution $v(w)$ depends continuously on $w$ and maps bounded sets to bounded sets. Our equation (12) now reduces to

$$
w=\mathscr{R}(I-P)(\nabla G(v(w)+w)+\varepsilon w-f),
$$

where $\mathscr{R}$ is the inverse of $\left.\tilde{\square}\right|_{N(\tilde{\square})^{\perp}}$. As we commented earlier, $\mathscr{R}$ is compact and hence the right hand side of (14) defines a completely continuous mapping on $N(\tilde{\square})^{\perp}$. This equation will have a solution if we can deform the right hand side to a compact linear operator while retaining the a priori bounds (by degree theory as in Schwartz [15]). We can do this by deforming $\nabla G$ to $K$ (that is, by using the deformation $t K+(1-t) \nabla G$ ) and by deforming $f$ to zero. The equation corresponding to (13) will then be linear. Hence $v$ will depend linearly on $w$ and thus the equation corresponding to (14) will be linear. Moreover, the bound for $\left\|u^{\varepsilon}\right\|_{2}$ is easily seen to hold uniformly during the deformation. In addition, it is easy but tedious to see that $v(w)$ changes continuously during the deformation and the bound for $v$ holds uniformly in the deformation. (Note that, since $G$ is convex, $K$ is positive definite.) Hence we finally see that (12) has a solution for all $f$ in $L^{2}(\Omega)^{n}$.

\section{REFERENCES}

[1] H. Amann, On the unique solvability of semilinear operator equations on Banach spaces, J. Math. Pures et Appl., 61 (1982), 149-175.

[2] P. Bates, Solutions of nonlinear elliptic systems with mixed spectra, Nonlinear Analysis, 4 (1980), 1023-1030.

[3] P. Bates and A. Castro, Existence and uniqueness for a variational hyperbolic system with resonance, Nonlinear Analysis, 4 (1980), 1151-1156.

[4] H. Brezis and L. Nirenberg, Forced vibrations of a nonlinear wave equation, Comm. Pure Applied Math., 31 (1978), 1-30.

[5] F. E. Browder, Non-solvability and the Fredholm alternative for mappings onto infinite-dimensional manifolds, J. Functional Anal., 8 (1971), 250-274.

[6] E. N. Dancer, Non-uniqueness for non-linear boundary-value problems, to appear.

[7] N. Dunford and J. T. Schwartz, Linear Operators, Part I, Interscience, New York, 1958.

[8] A. Friedman, Partial Differential Equations, Holt, Reinhart and Winston, New York, 1969.

[9] T. Kato, Perturbation Theory for Linear Operators, Springer-Verlag, Berlin, 1976.

[10] T. Kato, A generalization of the Heinz inequality, Proc. Jap. Acad., 6 (1961), 305-308.

[11] A. Lazer, Application of a lemma on bilinear forms to a problem in nonlinear oscillations, Proc. Amer. Math. Soc., 33 (1972), 89-94.

[12] J. Mawhin, Conservative systems of semilinear wave equations with periodic Dirichlet boundary conditions, J. Differential Equations, 42 (1981), 116-128.

[13] J. Mawhin and J. Ward, Nonresonance and existence for nonlinear elliptic boundaryvalue problems, Nonlinear Analysis, 6 (1981), 677-684. 
[14] C. Morrey, Multiple Integrals in the Calculus of Variations, Springer-Verlag, Berlin, 1966.

[15] J. T. Schwartz, Nonlinear Functional Analysis, Gordon and Breach, New York, 1969.

Received November 17, 1982.

UNIVERSITY OF NEW ENGLAND

ARMIDALE

N. S. W. 2350

AUSTRALIA

AND

RUTGERS UNIVERSITY

NEW BRUNSWICK, NJ 08903 


\title{
PACIFIC JOURNAL OF MATHEMATICS \\ EDITORS
}

\author{
DoNALD BABBITT (Managing Editor) \\ University of California \\ Los Angeles, CA 90024 \\ J. DugunduI \\ University of Southern California \\ Los Angeles, CA 90089-1113 \\ R. FINN \\ Stanford University \\ Stanford, CA 94305 \\ HERMANN FlasChKa \\ University of Arizona \\ Tucson, AZ 85721
}

C. C. Moore

University of California

Berkeley, CA 94720

ARTHUR OGUS

University of California

Berkeley, CA 94720

Hugo Rossi

University of Utah

Salt Lake City, UT 84112

H. SAMELSON

Stanford University

Stanford, CA 94305

ASSOCIATE EDITORS
R. ARENS
E. F. BECKENBACH
B. H. NeUMANN
F. WOLF
K. YoSHIDA (1906-1982)

\section{SUPPORTING INSTITUTIONS}

UNIVERSITY OF ARIZONA
UNIVERSITY OF BRITISH COLUMBIA
CALIFORNIA INSTITUTE OF TECHNOLOGY
UNIVERSITY OF CALIFORNIA
MONTANA STATE UNIVERSITY
UNIVERSITY OF NEVADA, RENO
NEW MEXICO STATE UNIVERSITY
OREGON STATE UNIVERSITY
UNIVERSITY OF ARIZONA
CALIFORNIA INSTITUTE OF TECHNOLOGY
UNIVERSITY OF CALIFORNIA
UNIVERSITY OF NEVADA, RENO
OREGON STATE UNIVERSITY
UNIVERSITY OF OREGON
UNIVERSITY OF SOUTHERN CALIFORNIA
STANFORD UNIVERSITY
UNIVERSITY OF HAWAII
UNIVERSITY OF TOKYO
UNIVERSITY OF UTAH
WASHINGTON STATE UNIVERSITY
UNIVERSITY OF WASHINGTON 


\section{Pacific Journal of Mathematics}

Vol. 115, No. $1 \quad$ September, 1984

Carlos Andradas Heranz and José Manuel Gamboa Mutuberría, A note on projections of real algebraic varieties $\ldots \ldots \ldots \ldots \ldots \ldots \ldots \ldots \ldots \ldots$

Jürgen Appell and Maria Patrizia Pera, Noncompactness principles in nonlinear operator approximation theory $\ldots \ldots \ldots \ldots \ldots \ldots \ldots \ldots \ldots \ldots$

Timothy John Carlson, Extending Lebesgue measure by infinitely many

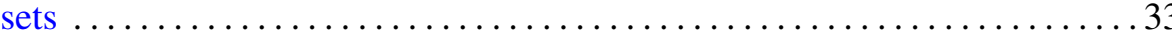

Donald S. Coram and Paul Frazier Duvall, Jr., Non-cell-like

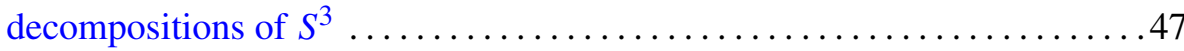

Edward Norman Dancer, Order intervals of selfadjoint linear operators and

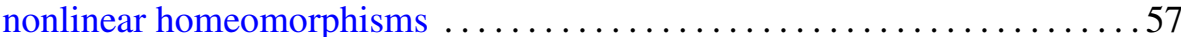

Ralph Jay De Laubenfels, Well-behaved derivations on $C[0,1] \ldots \ldots \ldots 73$

D. Feyel and A. de La Pradelle, Sur certaines extensions du théorème

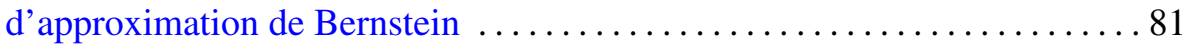

Colin C. Graham and Bertram Manuel Schreiber, Bimeasure algebras on

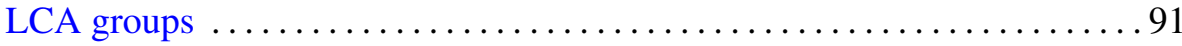

Richard Howard Hudson, Class numbers of imaginary cyclic quartic fields

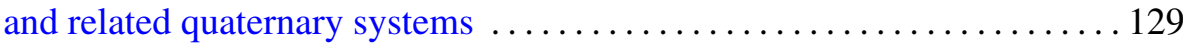

Carl Groos Jockusch, Jr. and Iraj Kalantari, Recursively enumerable sets and van der Waerden's theorem on arithmetic progressions . . . . . . . . 143

J. F. McClendon, On noncontractible valued multifunctions ........... 155 Akihiko Miyachi, Weak factorization of distributions in $H^{p}$ spaces $\ldots \ldots \ldots 165$ Ezzat S. Noussair and Charles Andrew Swanson, Global positive solutions of semilinear elliptic problems

Jon Christopher Snader, Strongly analytic subspaces and strongly

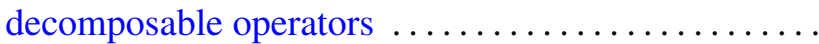

Boguslaw Tomaszewski, A construction of inner maps preserving the Haar measure on spheres

Akihito Uchiyama, The Fefferman-Stein decomposition of smooth functions and its application to $H^{p}\left(\mathbf{R}^{n}\right) \ldots \ldots \ldots$ 